\title{
eXpTools: A C++ class library for animation, tachistoscopic presentation, and response timing
}

\author{
C. BRUCE WARNER \\ Kutztown University, Kutztown, Pennsylvania \\ and \\ MICHAEL K. MARTIN \\ Naval Air Warfare Center, Training Systems Division, Orlando, Florida
}

\begin{abstract}
The eXpTools Library is a general-purpose tool for developing psychological experiments that combine animation with tachistoscopic presentation. The library's $\mathrm{C}++$ classes and assembly language functions are specialized for the creation of visual response time experiments. Its use is limited to variants of standard, 16-color, VGA high-graphics modes. However, it extends the capabilities of these modes through bit-plane animation techniques and a new, nonstandard, high-resolution graphics mode that will work with standard VGA cards and register-compatible cards. These techniques make possible a powerful animation class for managing complex animation or tachistoscopic presentations consisting of hundreds or thousands of frames. The library also combines such features as page flipping, screen blanking, video-refresh synchronization, interrupt-driven millisecond timing, interrupt-driven keyboard response collection, graphics primitives, bitmaps, and screen fonts. Utilities allow for the conversion of PCX graphics files and the creation of new screen fonts from monochrome bitmap files. The technologies and techniques underlying the library are presented along with an example program.
\end{abstract}

Research in cognition and perception often requires the precise temporal presentation of visual stimuli. Programming of experiments that require the apparent movement of visual stimuli is especially demanding, particularly when moving stimuli are to be temporally synchronized with the onset and offset of stationary stimuli. As an example, suppose you wish to conduct an experiment in which a trial begins with a preparatory message ("READY") appearing in the center of the screen for precisely $500 \mathrm{msec}$. After $500 \mathrm{msec}$, a stimulus array composed of moving and stationary elements is presented. The moving stimuli are to change position every $10 \mathrm{msec}$ so that a constant rate of movement is achieved (e.g., McLeod, Driver, \& Crisp, 1988, Experiment 1).

A simple, but unusable, approach would be to start a timer, write "READY" to the graphics screen, wait $500 \mathrm{msec}$, erase the message, reset the timer, write the stationary and moving stimuli to screen, poll the keyboard

Preparation of this manuscript was partially accomplished while the first author was supported by a grant from the Pennsylvania State System of Higher Education, Faculty Professional Development Council. The code and documentation in the eXpTools Library and the contents of this article represent solely the ideas of the authors and in no way represent the opinions of the U.S. Navy, the Naval Air Warfare Center, or Kutztown University. Portions of this article were adapted, with permission, from the eXpTools Library, copyright $1997-1999$ by C. Bruce Warner and Michael K. Martin. The authors thank reviewers Frank Bokhorst and Roger Graves for their helpful comments. M. K. Martin is now with the Logica Carnegie Group, Pittsburgh, PA. The authors dedicate this article to the memory of Montie McMickell. Correspondence should be addressed to C. B. Warner, Department of Psychology, Kutztown University, Kutztown, PA 19530 (e-mail: warner@kutztown.edu). and wait until $10 \mathrm{msec}$ have elapsed, erase the moving stimuli, reset the timer, write the moving stimuli to their new locations, and repeat this cycle of erasure, drawing, and keyboard polling every $10 \mathrm{msec}$ until the subject responds.

The well-known problem with this approach arises from the manner by which stimuli are displayed on a CRT. To display a stimulus, images are first written to the frame buffer of the video card. The images are then refreshed onscreen by an electron beam that scans the display from left to right and top to bottom. Without special precautions, a program's update of the image in video memory will not occur synchronously with the refresh cycle. If not synchronized, images can flicker and appear on screen disjointedly (shearing). Shearing and flicker are especially troublesome in animated sequences and are very noticeable by observers. Presentation timing errors, maximally equal to the refresh rate, can also occur (Creeger, Miller, $\&$ Paredes, 1990). Another problem with the procedure outlined above is that the subject may respond on the keyboard while the stimuli are being erased and redrawn. This would create response timing errors because the keyboard event would not be detected until the other processes are complete.

We will describe techniques for overcoming these technical difficulties and describe the freeware eXpTools Library, which was written over a number of years expressly to solve these problems. Many noncommercial solutions to problems of synchronization, tachistoscopic presentation, and timing (e.g., Creeger et al., 1990; Diesch, 1994; Dlhopolsky, 1988; Finley, 1991; Gabrielsson \& Jarvella, 1990; Graves \& Bradley, 1988; Paredes, Miller, 
\& Creeger, 1990; Segalowitz, 1987; von Brisinski, 1994) and many noncommercial solutions to problems of experiment generation (Eberhardt, Neverov, \& Haneef, 1997; Pallier, Dupoux, \& Jeannin, 1997) have been offered in recent years, but none of these solutions has focused specifically on combining tachistoscopic presentation with animation, the eXpTools Library's specialty.

\section{LIBRARY ORGANIZATION AND DESIGN}

The source code for the library is a mixture of $\mathrm{C}++$ and assembler. The full range of assembly language functions and $\mathrm{C}++$ classes within the library will not be described. (Numerous example programs are supplied with the library.) Functionally, however, the library can be divided into several parts. First, it supports screen management, including page flipping, refresh synchronization, screen blanking, and creation of bit-plane display pages. Second, it supplies classes for complex, multiple-framed, precision animation and tachistoscopic presentation, a set of millisecond and microsecond timing functions and timer classes, and a class for performing interrupt-driven response capture from the keyboard. Third, it supplies a keyboard class with key definitions. Fourth, the library supports graphics primitives (e.g., lines, rectangles, and ellipses), screen fonts (mono-spaced and proportionally spaced), and bit-mapped images (including monochrome and 16-color PCX files). Fifth, it supplies utilities for converting PCX graphics files to internal formats and utilities to generate new bit-mapped screen fonts.

In designing the eXpTools Library, we took an objectoriented approach to many of the components related to animation, tachistoscopic presentation, and response collection. We turned to object-oriented programming (OOP) in an attempt to control the complexity of the code otherwise required for even trivial animations or tachistoscopic presentations. Among the benefits ascribed to OOP are faster development, increased quality and maintainability, component reuse, enhanced code extensibility, and naturalness in modeling real-world systems (Booch, 1994; cf. Eberhardt et al., 1997). Software built in accordance with object-oriented principles achieves these advantages through encapsulation, abstraction, polymorphism, and inheritance of attributes (Coad \& Yourdon, 1991).

Encapsulation refers to the process of hiding the implementation details underlying a software module. Encapsulation is important because it reduces the complex interactions that could occur if programmers were free to access any of the variables and functions contained within a module rather than the select few designated by the designer of the module as the interface. Hence, programmers are encouraged to view software modules as interchangeable components that can be combined in various ways to produce complicated mechanisms.

In OOP, an object is a component with which the rest of the program will interact through the object's public interface. For example, in our design, the public interface for a sprite (moveable object) contractually obligates it to perform certain behaviors, such as drawing itself to the screen, erasing itself, and moving itself from one screen location to another. Although the operations required to draw text to the screen versus a circle to the screen differ considerably, the module that synchronizes presentation timing interacts only with the sprite interface and, hence, can operate unchanged with any object of the sprite type. Object abstraction of this type enhances the usability and extensibility of software components.

Most object-oriented languages achieve encapsulation and abstraction through the use of classes. In $\mathrm{C}++$, classes specify the structure, behavior, inheritance, and access permission for objects. Member variables provide the structure of the class, whereas member functions (methods) provide the behavior. Access permission is flexible in $\mathrm{C}++$. For example, private members may be accessed only by member functions of the class, but protected members may also be accessed by member functions of derived classes, and public members may be accessed by functions anywhere within the program. In carefully designed $\mathrm{C}++$ programs, member variables and functions are protected behind the public interface found within the class declaration.

Object-oriented languages such as $\mathrm{C}++$ achieve component reuse and extensibility through inheritance, in which the mechanisms of the base class or superclass are inherited by the derived class or subclass. The derived class can extend the behaviors of the base class by declaring new member variables and functions or can alter the behaviors of the base class by overriding its functions. Thus, in OOP, the software designer can create a hierarchy of classes in which the behaviors common to all objects of the class hierarchy are defined by the base classes, and specialized behaviors are defined by the derived classes. This "is-a-kind-of" relationship inherent in class hierarchies encourages software designers to model class relationships after systems in the real world that can also be described as hierarchical collections of properties and behaviors.

Several of the classes in the eXpTools Library (such as the sprite classes) are polymorphic in that they share an identical interface, which was inherited from the base class, but differ in the details of how the behaviors specified by the interface are implemented. In $\mathrm{C}++$, polymorphism is accomplished through pure virtual methods declared in the base class. Classes containing pure virtuals, known as abstract classes, cannot be instantiated; however, they can decree the general behaviors that derived classes must implement.

Although many components of the eXpTools Library take an object-oriented approach, others take a functionoriented approach. This is not so much a design flaw as it is a consequence of the fact that the core of the library was originally written in $\mathrm{C}$ and assembly language and evolved as $\mathrm{C}++$ compilers become available. Unlike a library for a pure, object-oriented language, such as Java, the eXpTools Library mirrors the mixed function- and objectoriented approach of $\mathrm{C}^{++}$. 


\section{SCREEN MANAGEMENT}

Precision animation or tachistoscopic presentation requires several basic building blocks. First, at least two relatively high resolution graphics pages must be available for smooth animation. Although tachistoscopic presentation may seem to require more than two display pages, the eXpTools Library provides $\mathrm{C}++$ animation classes that will perform most high-resolution, multiframed tachistoscopic presentations using only two display pages. Second, programs must have a means of synchronizing to the vertical refresh signal to avoid flicker and shearing. Third, keyboard responses must be collected even when the presentation program is busy drawing to screen. Finally, a millisecond or submillisecond timer is needed for timing the responses. The following sections describe the building blocks that will be used by the animation classes.

\section{High-Resolution Modes \\ Available on the Standard VGA}

To perform smooth, flicker-free animation, graphics programmers employ a technique known as double buffering, or page flipping. Segalowitz (1987) described a simple example of the technique. In color text modes, video memory is divided into as many as eight pages, only one of which is visible at once. The other pages can hold stimuli, however, and one can switch to these hidden pages tachistoscopically.

Creeger et al. (1990) and Paredes et al. (1990) applied the page-switching technique to graphics modes. Although suitable for text experiments and many experiments using graphics, the technique is still limited. The limitation has to do with resolution in standard VGA graphics modes. In text modes, at least four pages are always available; however, in graphics modes on standard VGA, the highest resolution one can obtain with four pages is the 16-color, $640 \times 200$ pixel mode $0 \times 0 \mathrm{E}$. Two pages are available in 16 -color, $640 \times 350$ pixel mode $0 \times 10$, and only one page in 16-color, $640 \times 480$ pixel mode $0 \times 12$.

The eXpTools Library does not support super VGA modes, but it does fully exploit and extend the highresolution video modes available on the standard VGA. The standard graphics modes supported by the eXpTools Library are MODE640X350 (BIOS mode 0x10) and MODE640X480 (BIOS mode $0 \times 12$ ). A nonstandard 16 color mode, MODE640X400, is also supported. This mode is created using a technique described by Abrash (1995) that increases the 350 vertical scan lines of MODE640X350 to 400 . The new mode, which does not have a standard VGA BIOS equivalent, is created by reprogramming the CRT controller to display the higher vertical resolution. MODE640X400 provides nearly as much vertical resolution as possible on the standard VGA, while giving the user two display pages. As a bonus, the new mode retains the higher $70-\mathrm{Hz}$ vertical refresh rate of MODE640X350, allowing more precise temporal control. In addition, all three of the previous modes can be modified by bit-plane management techniques, described in the next section, to increase the number of available display pages.

\section{Bit-Plane Management}

MODE640X480 provides the highest resolution available on the standard VGA at the expense of the multiple display pages needed for animation. If one is willing to sacrifice the number of colors simultaneously available on screen, the architecture of the VGA suggests a solution to this dilemma. The general technique has been described earlier by several authors (e.g., Abrash, 1995; Bokhorst, 1995; Wilton, 1987), but our implementation was developed independently, so we will describe some aspects of it in detail.

In MODE640X350, MODE640X400, and MODE$640 \mathrm{X} 480$, video memory is arranged into four $64 \mathrm{~K}$ bitplanes. Each plane occupies the same range of addresses at segment 0xA000. A bit-plane contains 1 bit for each pixel. A pixel is formed by combining 4 bits, 1 from each of the planes. There are 8 pixels per byte. Since the bitplanes occupy the same addresses, the microprocessor cannot access them directly. Instead, it must go through the video hardware. The video logic circuits, controlled by the microprocessor, determine which bit-planes are written to and read from. Since each bit-plane can be enabled and disabled independently, four two-color (monochrome) pages could be formed, each page having the same pixel resolution as the host video mode. Alternatively, using the planes in banks of two would yield two four-color bit-plane pages, the four colors being the background color plus three foreground colors. When combined with true page flipping in MODE640X350 and MODE640X400, up to eight monochrome bit-plane pages or four, four-color, bit-plane pages are possible.

A minor difficulty in using bit-planes as display pages stems from the fact that, normally, each plane codes a different attribute of the color image. The first three planes code values of blue (plane 0 ), green (plane 1), and red (plane 2), whereas the fourth plane (3) codes intensity. Used together, these 4 bits allow up to 16 colors on screen simultaneously. The solution, in the case of four monochrome bit-plane pages, is to reprogram the VGA Palette Registers with identical indices into the VGA DAC RAM so that colors do not shift when bit-planes are switched (for details, see Bokhorst, 1995, or Warner \& Martin, 1997). A similar adjustment can be made for four, fourcolor bit-plane pages.

The greatest difficulty in using bit-planes as display pages arises wher images are to be placed on the bitplane pages. To understand the difficulty, one must examine the flow of information in the VGA as images or graphics primitives are drawn to screen. Figure 1 shows the flow of information in VGA write mode 0. For example, when a byte of an image is being transferred to the display buffer, the byte is routed to the Arithmetic Logic Units (ALUs) corresponding to each bit-plane. The ALUs perform logical operations on the CPU data byte and the data bytes in the display latches, which are filled whenever a 


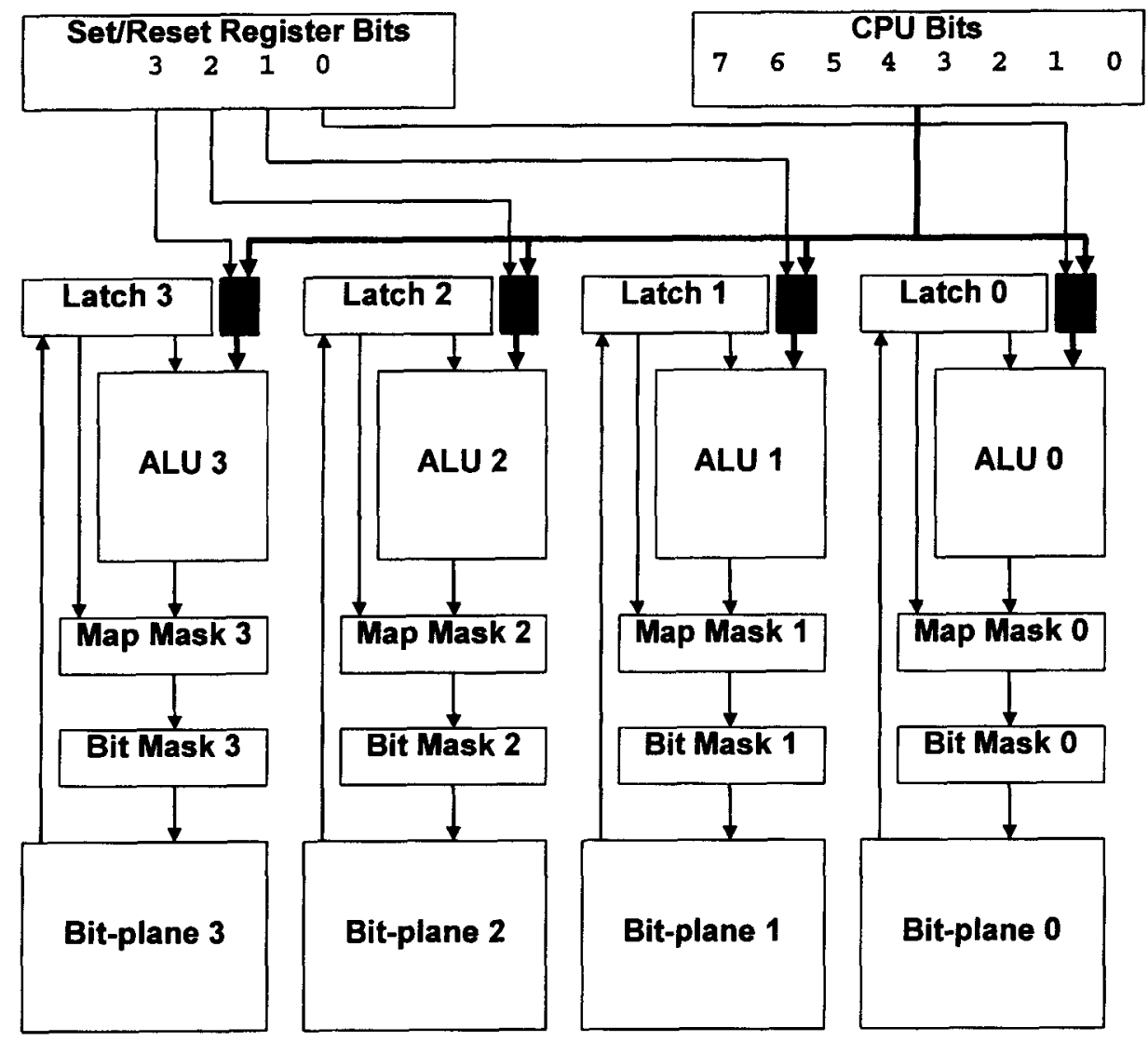

Figure 1. Position of the Map Mask Registers in the information flow of VGA write mode 0. From the eXpTools User's Manual by C. B. Warner and M. K. Martin, 1997. Copyright 1997-1999 by C. Bruce Warner and Michael K. Martin. Adapted with permission.

read operation takes place. Alternatively, the data byte for each bit-plane can come from the Set-Reset Register, which expands each of the 4 bits passed to it by the CPU into a full byte that can be passed to the corresponding bitplane. Since it is not always desirable to write to all four bit-planes simultaneously, a mechanism must exist to direct the data byte to the correct bit-plane. The Map Masks perform this function by preventing writes to bit-planes that are supposed to be preserved. The Map Mask (or Color Plane Enable) Register, which is at index 2 of the Sequencer, is the interface for the Map Masks. The lower 4 bits of this register control the status of the Map Masks. For instance, if the bits 0011 are written to this register, bit-planes 3 and 2 will be disabled for writing, and bitplanes 1 and 0 will be enabled for writing. The Bit Masks control which pixels within the byte of display memory being written to are modified.

The problem arises when functions from popular graphics libraries (such as Borland's BGI) are called on to draw images on the selected plane (or planes), since these functions typically alter the setting of the Map Mask Register. Inadvertently, these functions and other graphics functions disrupt the user's current bit-plane settings. Without preservation of the Map Mask Register, bit- planes are virtually useless as display pages. The eXpTools Library provides numerous graphics functions, including text, lines, fills, ellipses, rectangles, and bitmaps, that make bit-plane pages practical. All of these routines preserve the settings of the Map Mask Register. Generally, low-level details of bit-plane management, such as selecting and switching bit-planes, will be left to the Animator and AnimController classes (described in a later section), but $\mathrm{C}++$ functions are provided for those who prefer a function-oriented interface.

\section{Retrace Synchronization}

The technique used by the eXpTools Library to achieve retrace synchronization is the standard polling of the Input Status 1 register, at 0x03DA, for the vertical retrace signal, which arises when the video card is moving the electron beam from the lower right edge of the display to the upper left edge of the display. When the video card has entered the vertical retrace period, during which time the screen is blanked, bit 3 of Input Status Register 1 will toggle from zero to one.

The eXpTools Library provides two functions, _WaitOnRetrace and Wait_N_Retraces(ushort numRetraces), to synchronize to the status change of bit 3 . The function 
_WaitOnRetrace first polls to discover if vertical retrace is already in progress. If so, the code waits until it ends and then synchronizes to the leading edge of the next vertical retrace period. In this way, any code that must execute during the vertical retrace period will have almost the full period to work with. _Wait_N_Retraces adds a little additional overhead so that it can wait a specified number of retraces, supplied as an unsigned short integer to the function.

Users must pay attention to two details related to the polling of the vertical retrace signal. First, most (if not all) VGA cards latch the new display page before the vertical retrace begins. If the page switch is not programmed prior to the next pending vertical retrace period, timing will be off by one screen refresh cycle. Second, if a bitplane page is to be switched, the switch should occur during the vertical retrace period, since the change of bitplanes occurs immediately.

\section{Timing Responses}

The standard clock on PCs ticks every $54.9 \mathrm{msec}$. This resolution is too coarse for reaction time experiments, but special techniques can improve the resolution to $1 \mathrm{msec}$ or finer. Many solutions have been offered for improving the resolution without special hardware (e.g., Creeger et al., 1990; Dlhopolsky, 1988; Emerson, 1988; Graves \& Bradley, 1988; Heathcote, 1988). The Creeger et al. solution, which is similar to a technique published earlier by Willen and Krantz (1987), is the primary technique for the eXpTools Library. It forces the standard clock to tick approximately 1,000 times per second rather than its usual 18.2, yielding a resolution of $1 \mathrm{msec}$. Although simple to implement, the technique has two limitations: (1) It does reduce the available processing bandwidth due to the necessity of servicing the timer interrupt with each tick, ${ }^{1}$ and (2) the accuracy of the timer may be no better than 2 msec (Graves \& Bradley, 1988). The main advantage of the technique is that individual counters can be read and reset without using many processor clock cycles, which is why we chose it for use with the Animator class (described in a later section).

Abrash (1990) introduced a technique for microsecond timing, which is often used for timing small sections of code. The technique involves reprogramming the PC's $8253 / 4$ timer (or equivalent) so that it counts down cyclically from $0 \times 0000$ through $0 \times F F F F$ to $0 \times 0001$, at which point the timer interrupt is triggered. The resolution of the Abrash timer is $0.8381 \mu \mathrm{sec}$, so events can be timed with great accuracy, but, unfortunately, because each counter in the $8253 / 4$ is only 16 bits wide, the technique cannot handle time periods greater than 65,536 ticks (about $54.9 \mathrm{msec}$ ). To handle longer time periods, Abrash modified the technique to keep track of how many times the $8253 / 4$ had rolled over during the period to be timed. His code accomplished this by tracking the BIOS timer tick count, which increases by one every time the $8253 / 4$ count reaches $0 \times 0001$.
The most serious shortcoming of Abrash's second technique as a reaction timer or code timer is that occasional timing errors of up to $54.9 \mathrm{msec}$ can result (Dawdy, 1996). These errors occur because the BIOS count is not updated synchronously with the $8253 / 4$ timer, and, when the $8253 / 4$ timer is read near the rollover threshold, the interrupt handler may not have yet updated the BIOS count. The resulting read can be off by as much as 65,535 ticks. Many of the noncommercial implementations of this technique appear to contain this flaw. Dawdy (1996) offered a remedy based on checking for pending timer interrupts and adjusting the software count based on the impending interrupt status. We could not use his technique as published, because it requires the hardware counter to be reset after each read, which limits the number of counters present simultaneously to one.

Since we required multiple counters, we developed our own solution, which is based on two principles. First, the hardware counter need not be zeroed before making each measurement, as in the implementations of Abrash (1990) and Dawdy (1996). Instead, we can read the counter at both event start and stop and obtain the event duration through subtraction. This eliminates one of the main sources of error. Second, if a read occurs near the rollover threshold, we can save the value of the hardware counter and simply wait for the software counter to be updated before returning with the combined count. On installation, our microsecond timer determines the time required to service the timer interrupt, which is typically no more than a few microseconds. If a read occurs near the rollover threshold, our timer functions wait for the empirically determined duration before combining the hardware and software counts. Our solution appears to work well on a variety of different machines.

The $\mathrm{C}++$ wrapper class for the millisecond assembly language functions is MsecTimer, of which one global instance (Timer) exists within a given program. Up to eight 32-bit counters that count either up or down can be maintained. A ninth counter is used internally by eXpTools. The Timer object takes care of several details automatically. First, the eXpTools timer interrupt handler calls the default BIOS handler every $54.9 \mathrm{msec}$ to maintain time of day services and floppy disk motor timing. Second, since the timer interrupt handler exists within the code segment of the program that uses it, the millisecond timer must be removed and the original handler reinstated before the program terminates. Otherwise, when a new program is loaded into memory, the millisecond timer code will be overwritten, resulting in system failure when interrupt vector 8 is called. The Timer destructor ensures proper clean-up on program exit.

The microsecond assembly language functions are encapsulated by a hierarchy of $\mathrm{C}++$ classes. Each class is specialized for a different timing task, such as response timing versus code timing. The microsecond response timing class, $\mu$ secResponse Timer, maintains the count with quadword precision. Its accuracy is $\pm 0.8381 \mu \mathrm{sec}$ (as- 

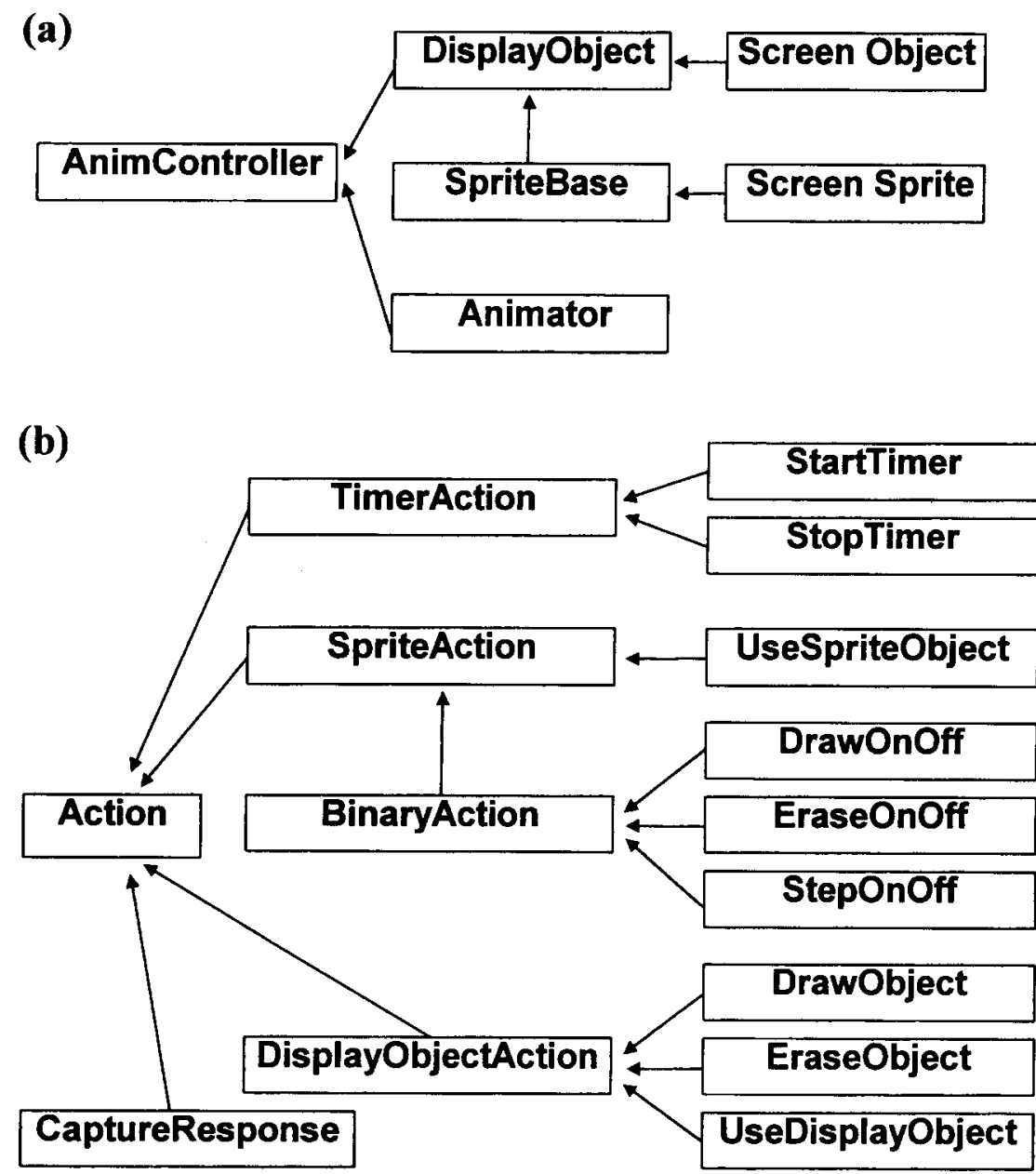

Figure 2. (a) The hierarchy of objects derived from AnimController. Static screen objects are derived from the abstract class DisplayObject, whereas sprites are derived from the abstract class SpriteBase. (b) The hierarchy of objects derived from the abstract class Action.

suming an accurate time base for the 8253/4), and there is no limit on how many timers are present at once. As with the MsecTimer class, the microsecond timer classes automatically take care of installation and deinstallation duties.

\section{Capturing Keyboard Responses}

The eXpTools Library includes a keyboard interrupt class, KeyInterrupt, which will capture keypresses and releases as they are signaled to the computer. This class allows keyboard responses to be recorded even if the program is drawing graphics or performing other tasks. As a subject responds on the keyboard, each keypress and release generates interrupt 9 . The default handler for this interrupt reads port $0 \times 60$, translates the 1 -byte scan codes found there to 2-byte codes, and stores them in low memory at $0 \times 41 \mathrm{E}$. The scan codes, after BIOS processing, uniquely identify each key. By replacing the default key handler with a customized version, responses can be cap- tured as soon as the CPU is interrupted. Some keys on the enhanced keyboard emit several 1-byte scan codes when a key is pressed. For response time collection, however, we need to know only the value of the first 1-byte code emitted by the keypress. If the subject is to respond on a specific key (or keys), as will be true of most experiments, the custom handler searches a table of candidate keys looking for a match. When a match is detected, the response time is read and stored for later use. Key releases can be captured and recorded in the same way.

Member functions are provided to install and remove the interrupt handler, return Response structures containing keystroke information, initiate and stop key capture, and set the various options for the class. For brevity, this code is not shown, but an example of usage of the class is shown in Listing 3. Only one global instance of the KeyInterrupt class (KeyHandler) is allowed to exist within a program. After capturing a keypress, KeyHandler's interrupt handler chains to the default handler so that normal 
BIOS keyboard services are maintained. Normally, the KeyHandler object is used in conjunction with the Animator class, which is described in the next section.

\section{ANIMATION CLASSES}

Animation or tachistoscopic presentation is implemented through the hierarchy of $\mathrm{C}++$ objects shown in Figure 2a. These classes are derived from AnimController, which controls the low-level details of doublebuffering and refresh synchronization. This class also keeps track of the current frame count of the animation, so that all derived classes have a common means of synchronizing their activities.

DisplayObject (Listing 1) is the abstract base class for static screen objects, such as bitmaps, lines, ellipses, or text, whereas SpriteBase (Listing 1) is the abstract base class for sprites, such as moving letters or moving line segments. To create a screen object or screen sprite, the programmer must derive a new object from either the DisplayObject or SpriteBase class and implement the essential behaviors of the object, such as drawing itself to screen, erasing itself from screen, moving itself from place to place, or changing its appearance.

The class Animator, whose public interface is shown in Listing 2 , is responsible for scheduling events and driving the event loop, described later in this section. Several kinds of events can be scheduled, including (1) timer events, such as starting or stopping a millisecond timer or initiating the capture of a keyboard response, (2) presentation of stationary screen objects, such as text, bitmaps, or graphics primitives, and (3) presentation of sprites, which are apparent motion stimuli. The unit of time used by the Animator class is the frame, which will vary from video mode to video mode but will always be a user-defined multiple of the video refresh period.

Internally, the Animator class controls framesynchronized events through the Action classes, shown in Figure $2 \mathrm{~b}$. The abstract base class of the Action hierarchy is Action. It declares a pure virtual member function called Act. Animator calls the virtual function Act at the count specified by the variable Count, which results in the derived version of Act being activated. Some of the derived classes start or stop timers, or start a response capture event, but most control the internal operation of DisplayObject- and SpriteBase-derived objects.

Animator automatically employs double-buffering and, in coordination with AnimController, takes care of all of the details of page flipping, bit-plane flipping, and event synchronization. In principle, Animator can display up to 65,535 frames, but, in practice, the number is limited by the available DOS memory for holding the events, display objects, sprites, fonts, and bitmaps required for a particular experiment. Although for most experiments this is a tremendous advance over previous methods of presentation, frame duration must be a multiple of the time taken to draw the most complex frame, which might prove unacceptable in some circumstances.
Internally, the Animator maintains events as ordered lists, sorted by frame count. Sprites and display objects are maintained as unordered lists. These lists are used by the event loop, which drives stimulus presentation. A schematic diagram of Animator's event loop is shown in Figure 3. When Animator::Animate is called, the event loop cycles through the events and sprites until the last frame has been displayed. During each cycle of the loop, four separate event lists are walked, and the sprite list is walked twice. Timer events are processed first, followed by display-object-erase events, sprite-erase events, useobject events (change of on-screen appearance; e.g., an " $X$ " to an " $O$ "), display-object-draw events, and spritedraw events. Terminal events, at the ends of the arrows, are usually made through the Action classes. Sprite erases and draws, however, are executed directly by the Erase and Draw members of the SpriteBase class.

The individual programming the experiment must create display objects and sprites that are compatible with Animator's internal mechanisms. To do so, the programmer must follow three simple rules: (1) display objects must provide member functions to draw and erase themselves and, optionally, change their on-screen appearance, if desired, (2) screen draws and erases must be accomplished as quickly as possible, and (3) sprites, in addition to supplying member functions for drawing and erasing themselves, must provide a member function to change to new screen locations and store the previous two positions for proper erasure of images at former locations. Detailed information on implementing these rules is available in the eXpTools manual.

To aid in the creation of complex animations, the programmer can link to a single-step version of the eXpTools Library. Using keystrokes, the programmer can step through the animation one frame at a time, verifying that each event appears at the scheduled frame. Animator member functions are also available to estimate the number of video refreshes required per frame, based on measurement of the actual time taken to draw each frame to screen.

\section{A SIMPLE EXAMPLE}

Imagine that we wish to present a moving monochrome bitmap on screen. Beginning with the 35 th frame, the bitmap is to move from the top of the screen toward the bottom for 380 frames, at the rate of 1 pixel per frame. The observer is to respond to the presence of the moving stimulus as rapidly as possible by pressing the spacebar key.

As shown in Listing 3, we will derive a new class, MonoBitmapSprite, from SpriteBase to draw the moving stimulus to screen. This class overrides the Step and Draw virtual members of SpriteBase so that Animator can direct MonoBitmapSprite to appear and change its location at the appropriate times. Animator will call the MonoBitmapSprite member functions Step, Draw, and Erase every frame. Notice that Step checks the variable OKToStep to ensure that it has permission to move from one location to another. Step also has the responsibility of storing the 


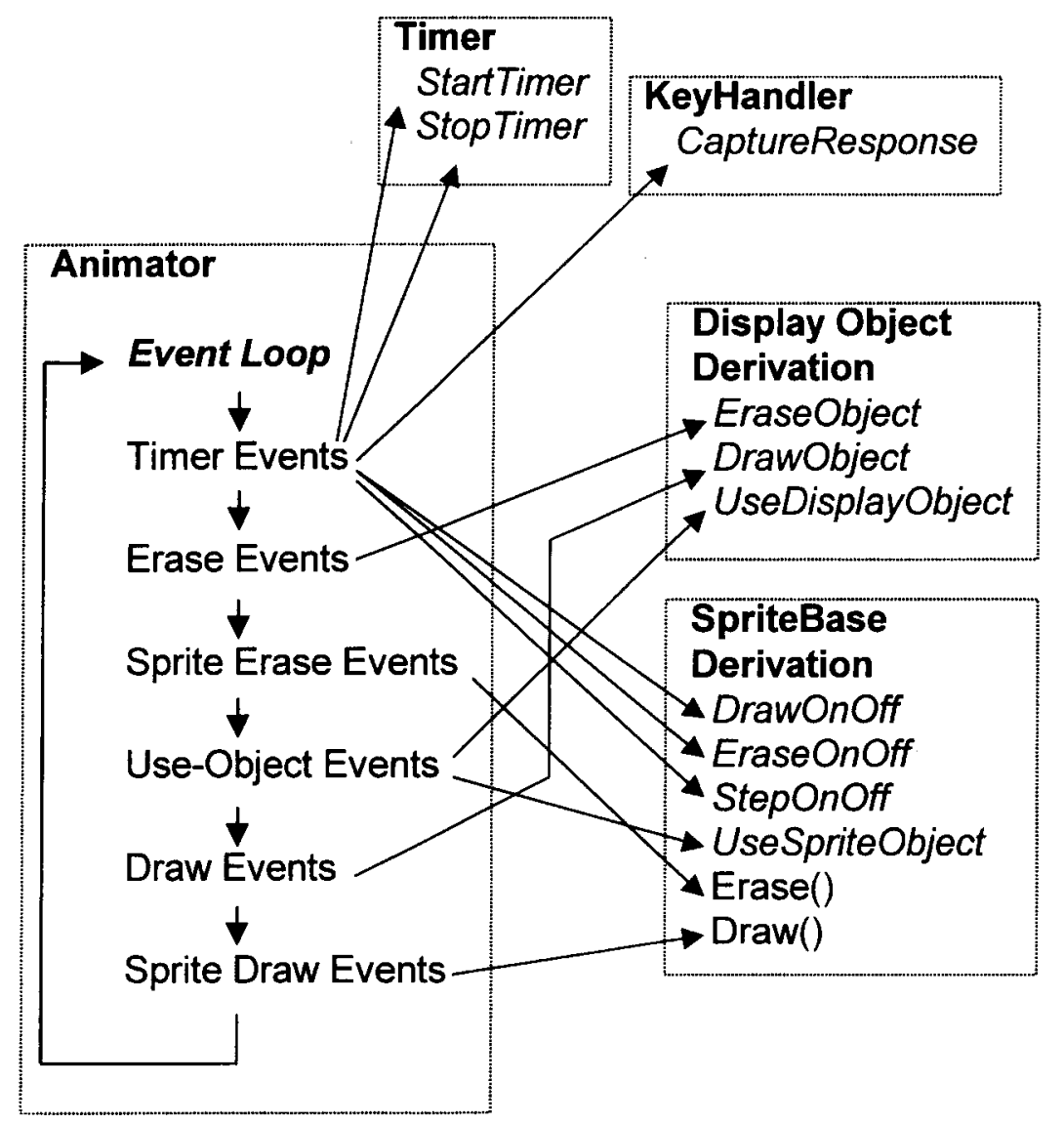

Figure 3. A schematic representation of Animator's event loop. Member functions written in italics are activated through the Action classes. The Draw and Erase member functions of SpriteBase-derived objects are called directly by Animator. From the eXpTools User's Manual by C. B. Warner and M. K. Martin, 1997. Copyright 1997-1999 by C. Bruce Warner and Michael K. Martin. Adapted with permission.

bounding box around the current location of the bitmap, so that it can be erased from the screen before the image is redrawn at the next screen location. Draw checks the variable OKToDraw to ensure that it has permission from Animator to appear on-screen during a given frame. Failure to check these variables will result in timing errors and anomalous behaviors. In this program, we had no reason to override Erase, because its default behavior, defined by SpriteBase (Listing 1), is satisfactory.

An object of the Animator class, anim, is created in function main to schedule and execute screen events and coordinate these events with response collection. The first parameter passed to the constructor is a constant, which specifies the number of refreshes per frame of the animation sequence. The second parameter is the animation mode. Next, the response key, space $(0 \times 39)$, is passed to SetNumResponseKeys. (The hexidecimal "make" code of the key was obtained from the eXpTools example program, KeySpy.)

To present an object derived from DisplayObject or SpriteBase, it must be registered with anim. An object of the MonoBitmapSprite class is created by operator new and passed to AddSprite(SpriteBase *psprite, uint count, uint duration), along with the starting count of presentation (in frames), and the on-screen duration (in frames). The call to Animate runs the trial sequence. Following the animation, Reset must be called to deallocate event lists within the anim object and to free the screen object assigned earlier to anim. Failure to do so will lead to erratic operation. The response itself is collected from the global keyboard interrupt object, KeyHandler, in the form of a structure containing three members: (1) ResponseCode, the index of the key that was pressed in the array of key codes passed to SetNumResponseKeys; (2) ResponseKey, the actual make or break code; and (3) RT, the response time in milliseconds. In this example, we used only RT.

\section{CONCLUSION}

The eXpTools Library provides many useful classes, functions, and utilities for the creation of psychological experiments, especially those that combine animation with tachistoscopic presentation. The Animator class simplifies the coordination of screen events by encapsulating the library's assembly language screen-management functions and hiding these details from the programmer. Although 
we employed a simple example that used the linear apparent movement of a single stimulus, multiple stimuli can be scheduled with Animator, and complex patterns of behavior can be programmed into SpriteBase derived objects.

\section{Known Limitations}

The graphics library supplied with eXpTools is limited to the 16 color modes listed earlier. Because it was originally designed to operate quickly on 286 machines, it does not take advantage of the capabilities of 386 and higher processors. On the other hand, it will run on the vintage machines that seem to exist in many departments (such as the first author's). The available graphics primitives are limited, but others could be added by a skilled programmer. Animator does not check for object collision, nor does it allow the programmer to set the order in which sprites and display objects are drawn to screen. This limitation again stems from our original need to have programs run efficiently on older, slower hardware. Some bugs may not yet have been identified because the library has not been subjected to much "real-world" stress. The keyboard interrupt class tends to interact with Turbo Debugger, sometimes resulting in a crash.

\section{Future Version}

The first author is developing an enhanced version of the library for Windows $95 / 98 / \mathrm{NT}$. It will be based on Microsoft DirectX technology. Neither an anticipated release date nor a feature list is available at the current time.

\section{Program Availability}

The entire package, with libraries for Borland $\mathrm{C}_{++}$, documentation, full $\mathrm{C}++$ and assembly language source code, example programs, utilities for converting PCX bitmap files, and utilities for creating screen fonts is available from the first author on three 1.44-MB disks. Each disk contains a self-extracting archive. Although use of the package is free, distribution is controlled. To obtain the software, send an e-mail to the first author at warner@ kutztown.edu requesting a copy. You will be e-mailed distribution details. Until the library is available by ftp, requesters will have to pay a small distribution fee to cover the cost of disks and postage, and the labor to copy and mail the disks (typically under $\$ 20$ if mailed in the United States). No technical support is available and the distribution fee is nonrefundable.

\section{REFERENCES}

AbRash. M. (1990). Zen of assembly language: Vol. 1. Knowledge. Glenview, IL: Scott-Foresman.

ABRASH, M. (1995). Zen of graphics programming. Scottsdale, AZ: Coriolis.

BOKHORST, F. D. (1995). Bit-plane layering for high-resolution EGA and VGA graphics on the IBM PC/XT/AT. Behavior Research Methods, Instruments, \& Computers, 27, 496-501.

Booch, G. (1994). Object-oriented analysis and design with applications (2nd ed.). Redwood City, CA: Benjamin/Cummings.

COAD, P., \& Yourdon, E. (1991). Object-oriented design and analysis (2nd ed.). Englewood Cliffs, NJ: Prentice Hall.
Creeger, C. P., Miller, K. F., \& Paredes, D. R. (1990). Micromanaging time: Measuring and controlling timing errors in computercontrolled experiments. Behavior Research Methods, Instruments, \& Computers, 22, 34-79.

DAWDY, M. (1996). A more accurate timing tool. Windows Developer's Journal, 7 [online]. Available: http://www.wdj.com/notgone/708art.htm

Diesch, E. (1994). A high-resolution color graphic display four-channel software tachistoscope. Behavior Research Methods, Instruments, \& Computers, 26, 331-334.

DLhopolsKy, J. G. (1988). C language functions for millisecond timing on the IBM PC. Behavior Research Methods, Instruments, \& Computers, 20, 560-565.

Eberhardt, S. P., Neverov, M., \& Haneef, O. (1997). RunScript: An extendable object-oriented program for computer-controlled psychology experiments. Behavior Research Methods, Instruments, \& Computers, 29, 313-321.

EMERSON, P. L. (1988). TIMEX: A simple IBM AT C language timer with extended resolution. Behavior Research Methods, Instruments, \& Computers, 20, 566-572.

FINLEY, G. P. (1991). A VGA tachistoscope. Behavior Research Methods, Instruments, \& Computers, 23, 546-547.

Gabrielsson, J., \& Jarvella, R. J. (1990). How to obtain nearmillisecond precision on the IBM PC with visual stimuli not exceeding one printed line. Behavior Research Methods, Instruments, \& Computers, 22, 520-525.

Graves, R., \& Bradley, R. (1988). More on millisecond timing and tachistoscopic applications for the IBM PC. Behavior Research Methods, Instruments, \& Computers, 20, 408-412.

HeATHCOTE, A. (1988). Screen control and timing routines for the IBM microcomputer family using a high-level language. Behavior $R e-$ search Methods, Instruments, \& Computers, 20, 289-297.

McLeod, P., Driver, J., \& CrisP, J. (1988). Visual search for a conjunction of movement and form is parallel. Nature, 332, 154-155.

Pallier, C., Dupoux, E., \& Jeannin, X. (1997). EXPE: An expandable programming language for on-line psychological experiments. $B e-$ havior Research Methods, Instruments, \& Computers, 29, 322-327.

Paredes, D. R., Miller, K. F., \& Creeger, C. (1990). Graphic precision: Controlling stimulus displays on IBM PC-compatible computers. $B e-$ havior Research Methods, Instruments, \& Computers, 22, 319-322.

Segalowitz, S. J. (1987). IBM PC tachistoscope: Text stimuli. Behavior Research Methods, Instruments, \& Computers, 19, 383-388.

VON BRISINSKI, I. S. (1994). Ultrafast display buildup with standard VGA on MS-DOS computers. Behavior Research Methods, Instruments, \& Computers, 26, 335-336.

WARNER, C. B., \& MARTIN, M. K. (1997). eXpTools User's Manual. (Available with eXpTools Library from C. Bruce Warner, Department of Psychology, Kutztown University Kutztown, PA 19530).

WILLEN, D. C., \& KRANTZ, J. I. (1987). 8088 assembler language programming: The IBM PC (2nd ed.). Indianapolis, IN: Sams.

WILTON, R. (1987). Programmer's guide to $P C$ and $P S / 2$ video systems. Redmond, WA: Microsoft Press.

\section{NOTE}

1. We measured the time required to process the interrupt service routine (ISR) of our millisecond timer on four machines: a $12-\mathrm{MHz} 286$ (286-12), a 66-MHz 486 (486-66), a 100-MHz Pentium (P5-100), and a 200-MHz AMD K6 (K6-200). With the default number of three counters installed, the 286-12 took a mean time of $29.05 \mu \mathrm{sec}$ to process the interrupt. The mean times required to update three counters on a 486-66, a P5100 , and a K6-200 were $4.27,1.88$, and $1.16 \mu \mathrm{sec}$, respectively. These figures predicted performance declines for the foreground process of $2.91 \%(286-12), 0.42 \%(486-66), 0.19 \%$ (P5-100), and 0.12\% (K6-200). However, since these estimates did not include the time required to reflect the interrupt to the ISR, we measured the actual percent reduction in performance for a simple program that performed computations in a loop. The mean percent reductions in the number of computations performed were $3.05 \%(286-12), 0.80 \%(486-66), 0.33 \%$ (P5-100), and $0.15 \%$ (K6200 ). The costs for each additional counter, up to eight, were $0.48 \%$ (28612 ), $0.036 \%$ (486-66), $0.015 \%$ (P5-100), and 0.0046\% (K6-200). 


\section{LISTING 1}

DisplayObject and SpriteBase: The Abstract Base Classes for Screen Objects

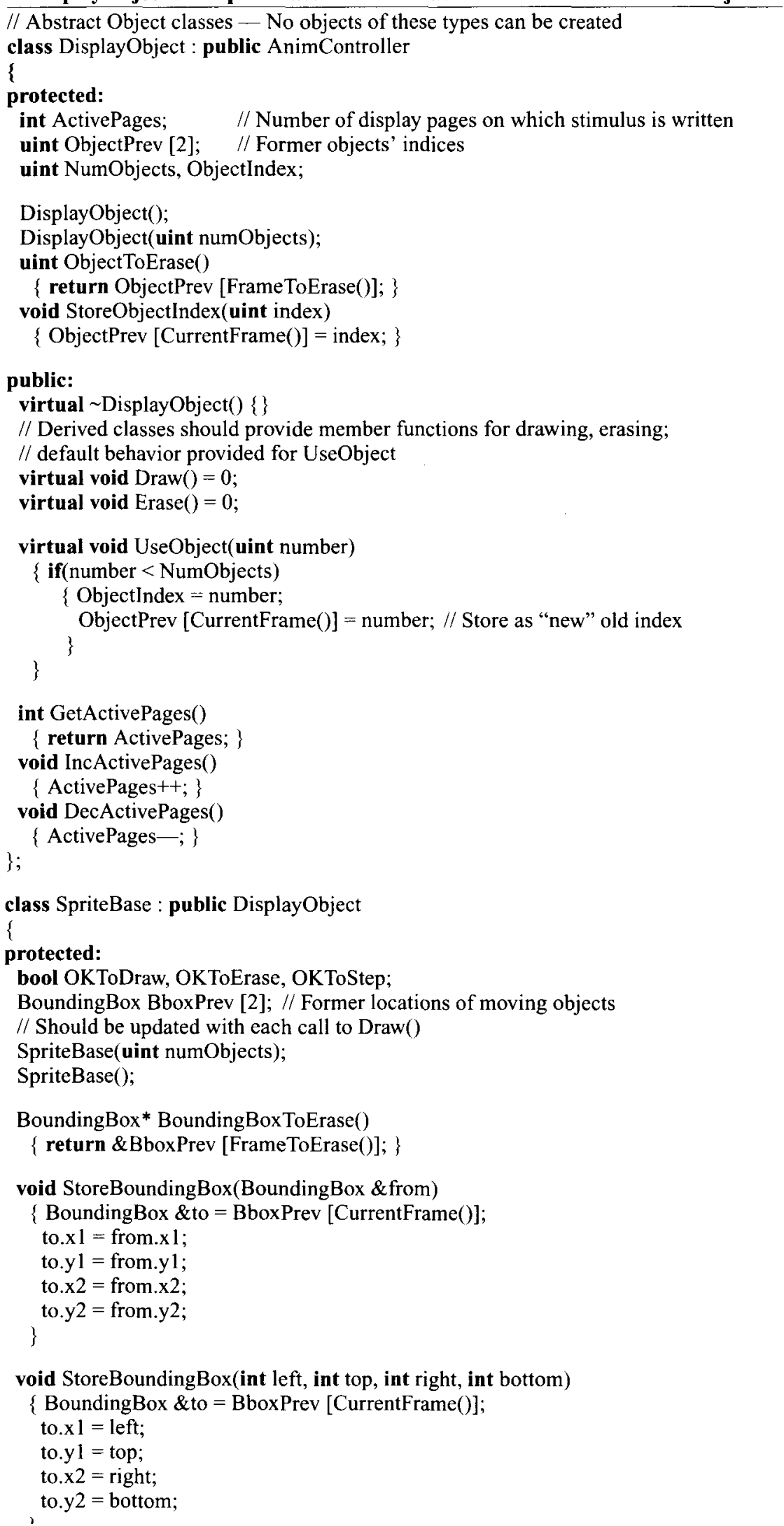




\section{LISTING 1 (Continued)}

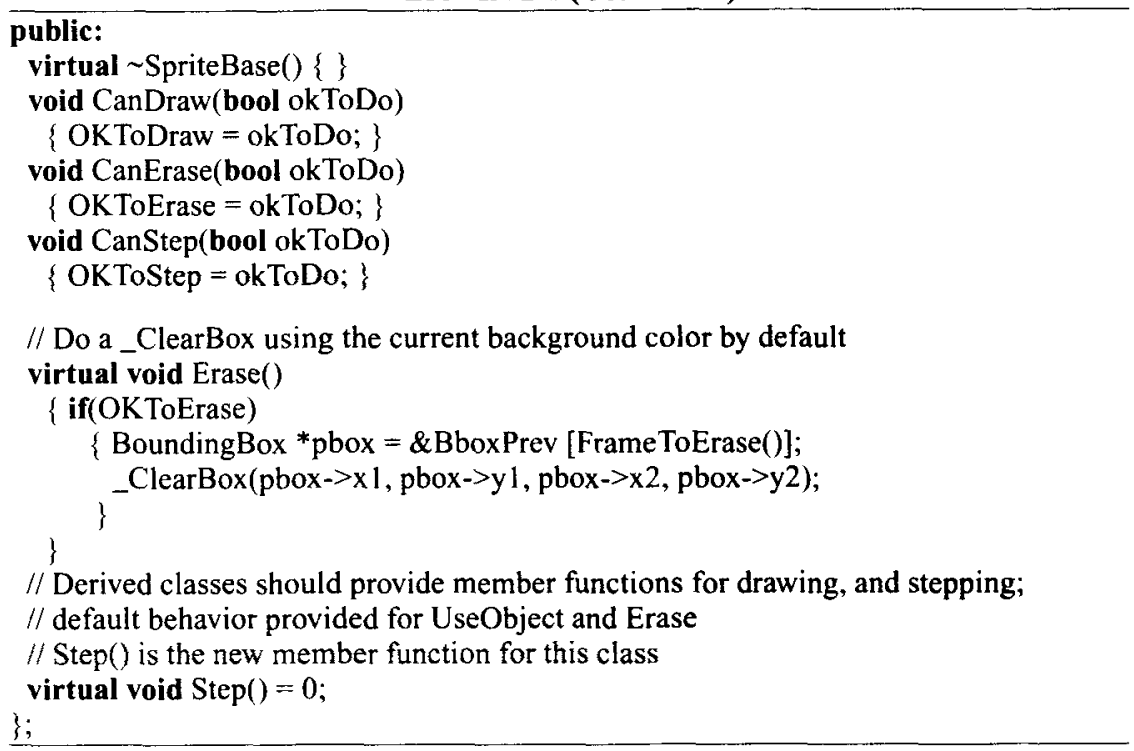

LISTING 2

Animator's Public Member Functions

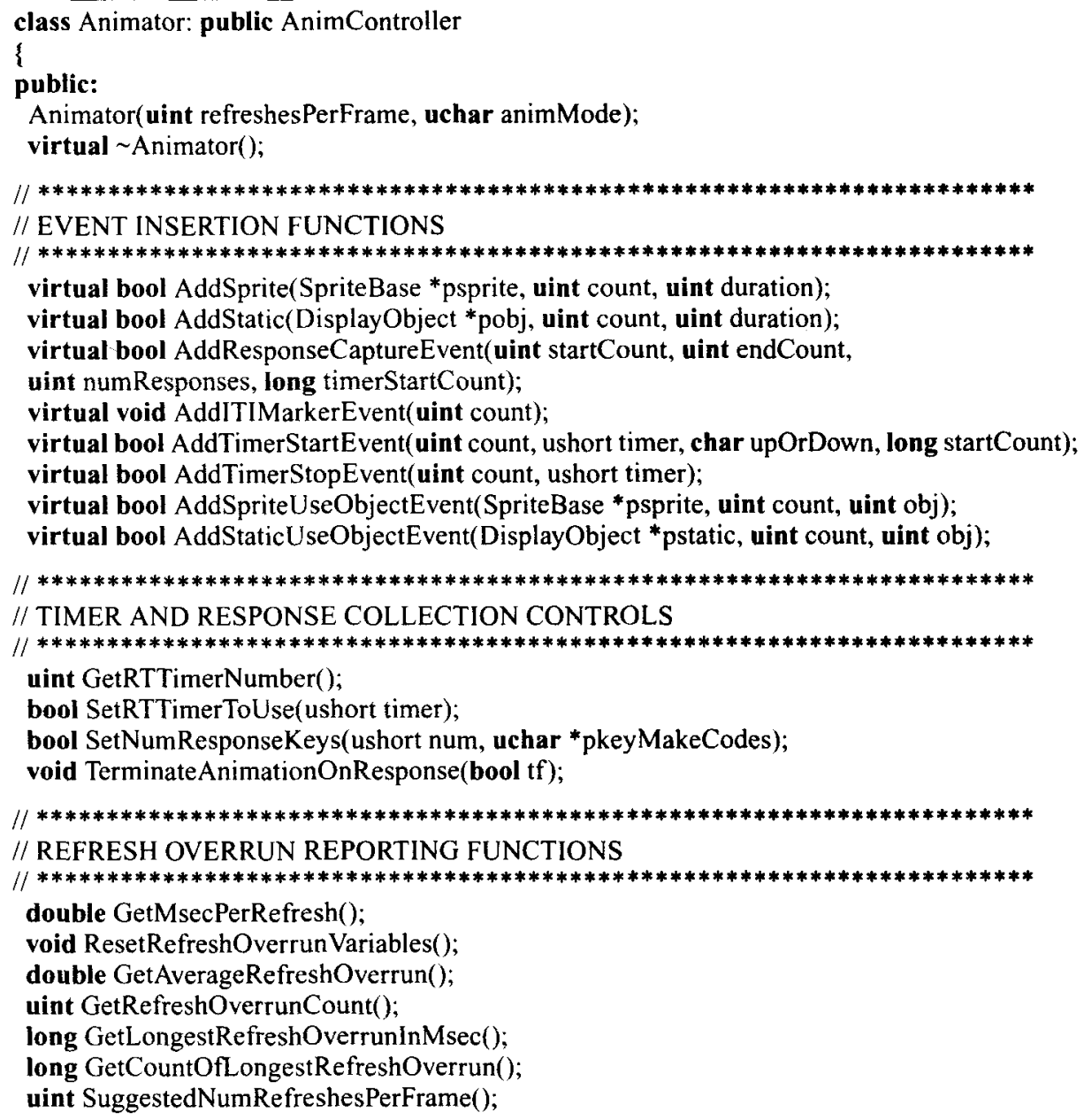


LISTING 2 (Continued)

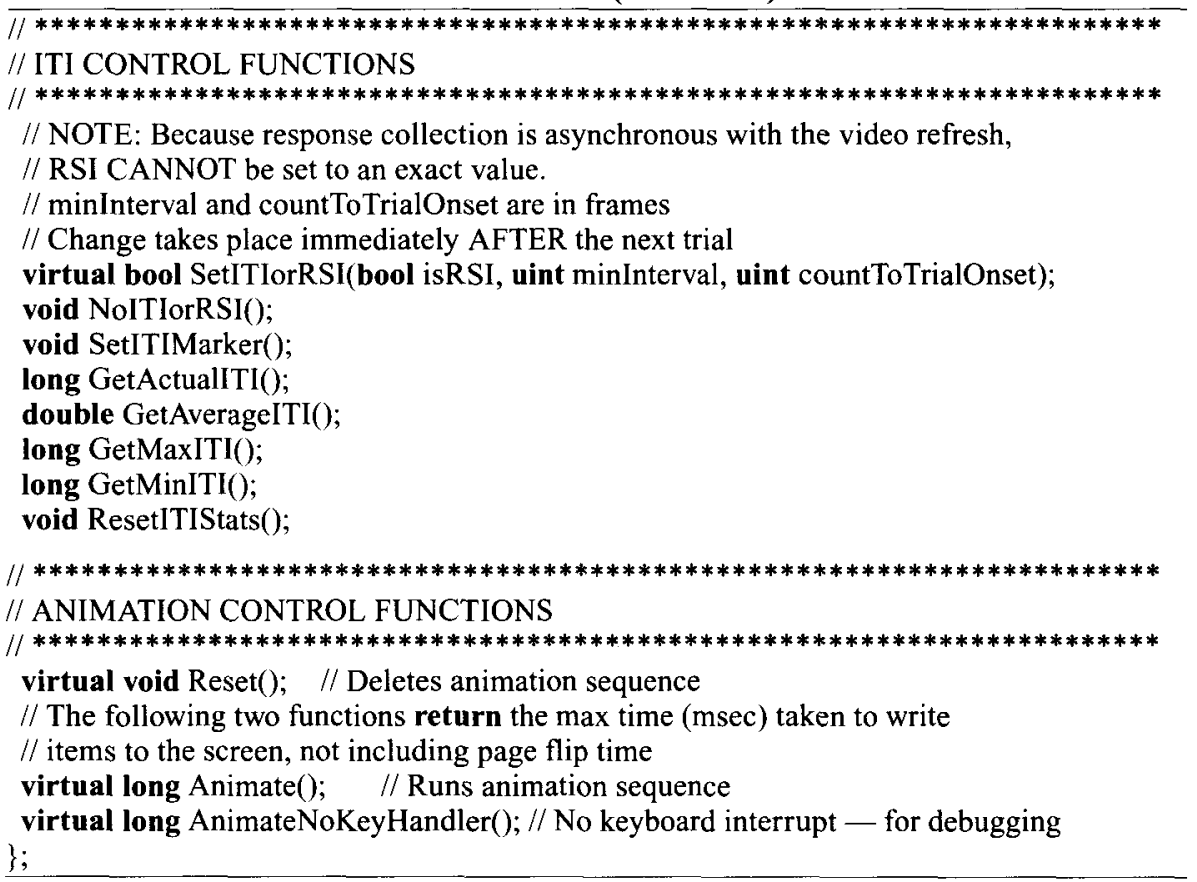

LISTING 3

A Simple Example Program

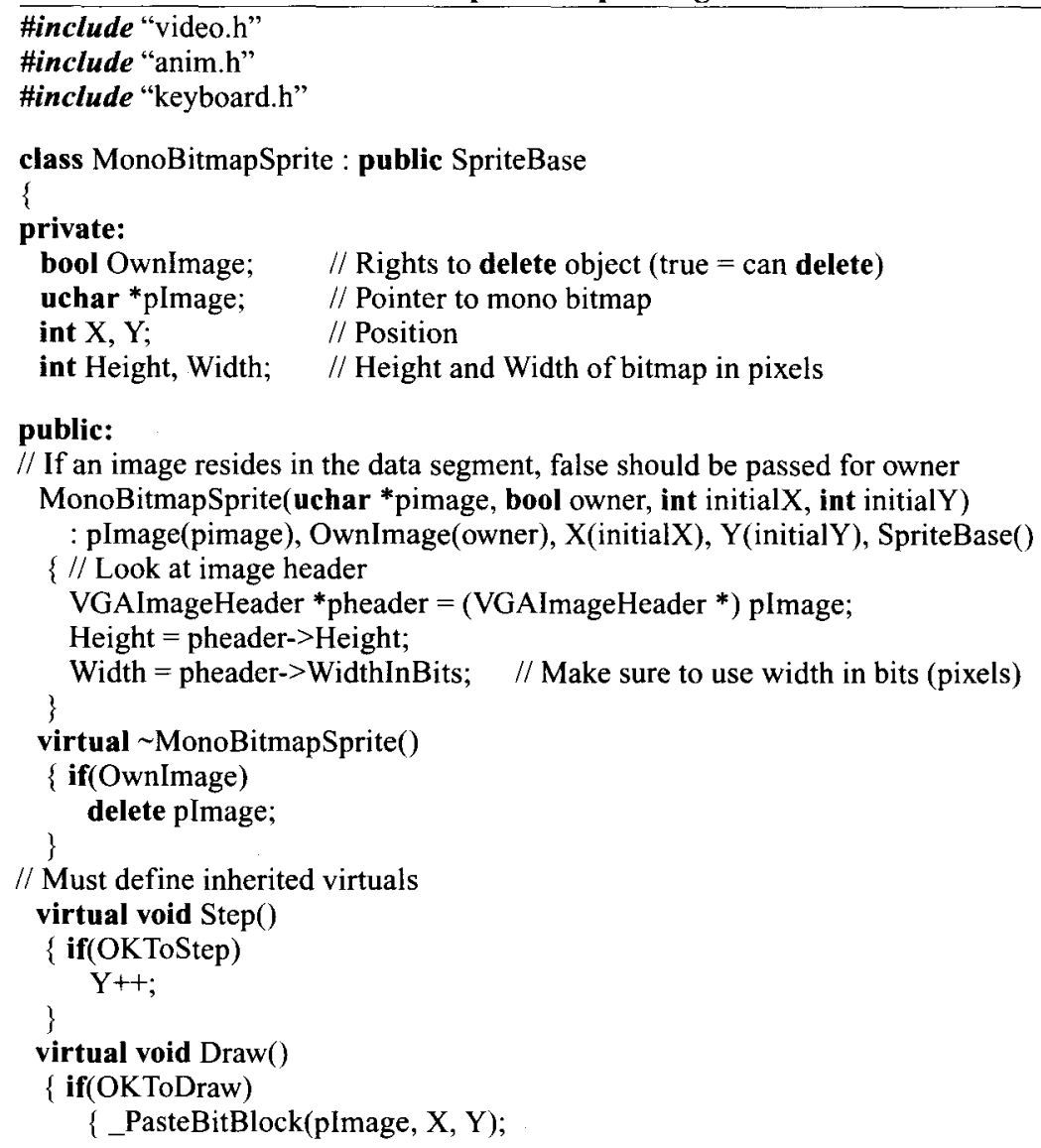


LISTING 3 (Continued)

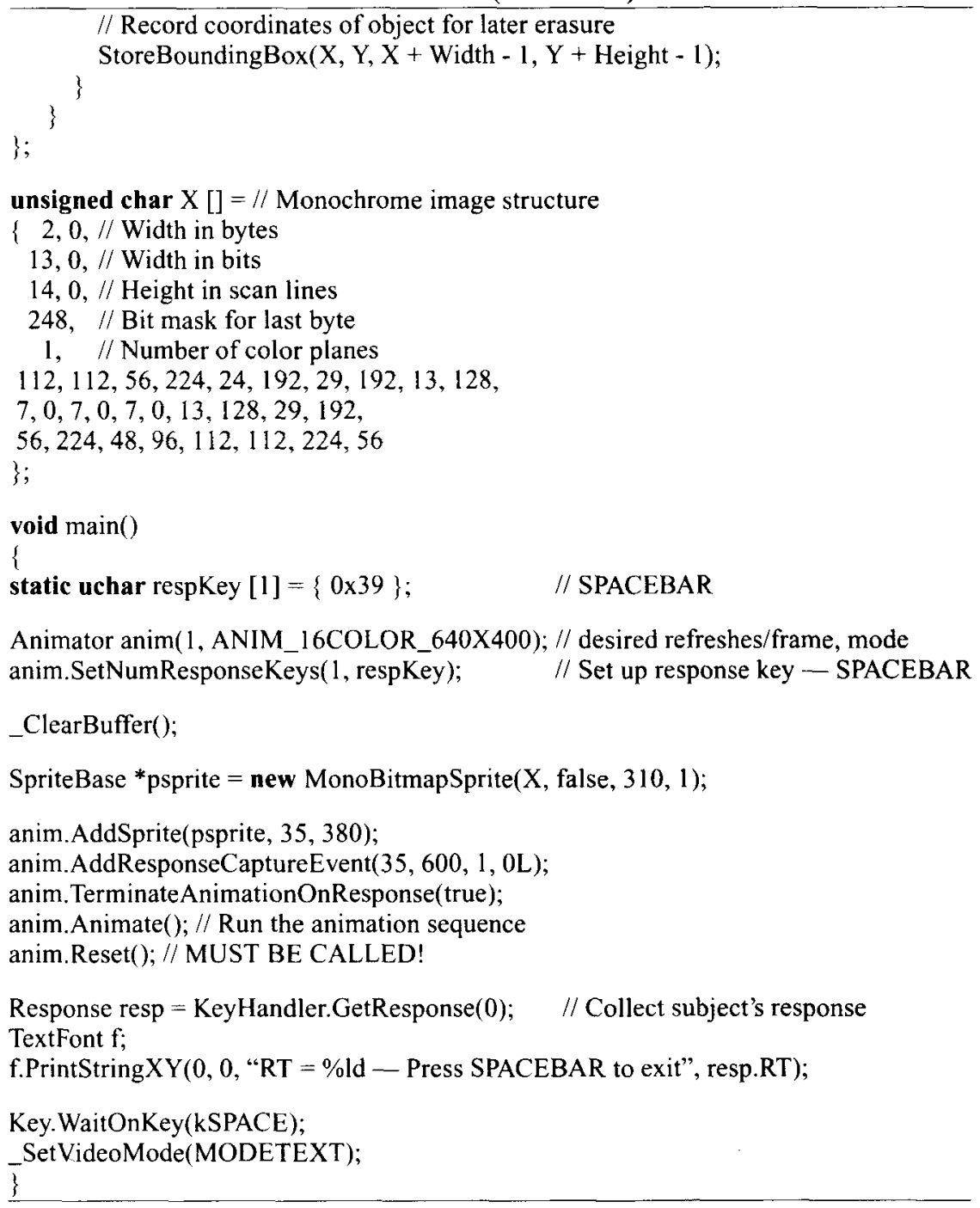

